\title{
Erratum: Genetic loci associated with heart rate variability and their effects on cardiac disease risk
}

Ilja M. Nolte et al. ${ }^{\#}$

\#A full list of authors and their affiliations appears at the end of the paper.

Nature Communications 8:15805 doi: 10.1038/ncomms15805 (2017); Published 14 Jun 2017; Updated 2 Aug 2017

In Supplementary Fig. 10 of this Article, images for panels a and b were inadvertently omitted. The correct version of Supplementary Fig. 10 is provided as Supplementary Information associated with this Erratum. Furthermore, the affiliation details for Azmeraw T. Amare, Benedikt von der Heyde, and Marcel den Hoed are incorrect in this Article. The correct affiliation details for these authors are given below.

Azmeraw T. Amare:

Department of Epidemiology, University of Groningen, University Medical Center Groningen, PO Box 30001, Groningen 9700 RB, The Netherlands.

School of Medicine, University of Adelaide, Adelaide, South Australia, SA 5005, Australia

College of Medicine and Health Sciences, Bahir Dar University, Bahir Dar 6000, Ethiopia.

Benedikt von der Heyde:

Department of Immunology, Genetics and Pathology, Medical Genetics and Genomics, Uppsala University, Uppsala 75327, Sweden.

Science for Life Laboratory, Uppsala University, Uppsala 75237, Sweden.

Marcel den Hoed:

Department of Immunology, Genetics and Pathology, Medical Genetics and Genomics, Uppsala University, Uppsala 75327, Sweden.

Science for Life Laboratory, Uppsala University, Uppsala 75237, Sweden.

\begin{abstract}
(c) (i) Open Access This article is licensed under a Creative Commons Attribution 4.0 International License, which permits use, sharing, adaptation, distribution and reproduction in any medium or format, as long as you give appropriate credit to the original author(s) and the source, provide a link to the Creative Commons license, and indicate if changes were made. The images or other third party material in this article are included in the article's Creative Commons license, unless indicated otherwise in a credit line to the material. If material is not included in the article's Creative Commons license and your intended use is not permitted by statutory regulation or exceeds the permitted use, you will need to obtain permission directly from the copyright holder. To view a copy of this license, visit http://creativecommons.org/licenses/by/4.0/
\end{abstract}

(C) The Author(s) 2017

Ilja M. Nolte, M. Loretto Munoz, Vinicius Tragante, Azmeraw T. Amare, Rick Jansen, Ahmad Vaez, Benedikt von der Heyde, Christy L. Avery, Joshua C. Bis, Bram Dierckx, Jenny van Dongen, Stephanie M. Gogarten, Philippe Goyette, Jussi Hernesniemi, Ville Huikari, Shih-Jen Hwang, Deepali Jaju, Kathleen F. Kerr, Alexander Kluttig, Bouwe P. Krijthe, Jitender Kumar, Sander W. van der Laan, Leo-Pekka Lyytikäinen, Adam X. Maihofer, Arpi Minassian, Peter J. van der Most, Martina Müller-Nurasyid, Michel Nivard, Erika Salvi, James D. Stewart, Julian F. Thayer, Niek Verweij, Andrew Wong, Delilah Zabaneh, Mohammad H. Zafarmand, Abdel Abdellaoui, Sulayma Albarwani, Christine Albert, Alvaro Alonso, Foram Ashar, Juha Auvinen, Tomas Axelsson, Dewleen G. Baker, Paul I.W. de Bakker, Matteo Barcella, Riad Bayoumi, Rob J. Bieringa, Dorret Boomsma, Gabrielle Boucher, Annie R. Britton, Ingrid Christophersen, Andrea Dietrich, George B. Ehret, Patrick T. Ellinor, 
Markku Eskola, Janine F. Felix, John S. Floras, Oscar H. Franco, Peter Friberg, Maaike G.J. Gademan, Mark A. Geyer, Vilmantas Giedraitis, Catharina A. Hartman, Daiane Hemerich, Albert Hofman, Jouke-Jan Hottenga, Heikki Huikuri, Nina Hutri-Kähönen, Xavier Jouven, Juhani Junttila, Markus Juonala, Antti M. Kiviniemi, Jan A. Kors, Meena Kumari, Tatiana Kuznetsova, Cathy C. Laurie, Joop D. Lefrandt, Yong Li, Yun Li, Duanping Liao, Marian C. Limacher, Henry J. Lin, Cecilia M. Lindgren, Steven A. Lubitz, Anubha Mahajan, Barbara McKnight, Henriette Meyer zu Schwabedissen, Yuri Milaneschi, Nina Mononen, Andrew P. Morris, Mike A. Nalls, Gerjan Navis, Melanie Neijts, Kjell Nikus, Kari E. North, Daniel T. O'Connor, Johan Ormel, Siegfried Perz, Annette Peters, Bruce M. Psaty, Olli T. Raitakari, Victoria B. Risbrough, Moritz F. Sinner, David Siscovick, Johannes H. Smit, Nicholas L. Smith, Elsayed Z. Soliman, Nona Sotoodehnia, Jan A. Staessen, Phyllis K. Stein, Adrienne M. Stilp, Katarzyna Stolarz-Skrzypek, Konstantin Strauch, Johan Sundström, Cees A. Swenne, Ann-Christine Syvänen, Jean-Claude Tardif, Kent D. Taylor, Alexander Teumer, Timothy A. Thornton, Lesley E. Tinker, André G. Uitterlinden, Jessica van Setten, Andreas Voss, Melanie Waldenberger, Kirk C. Wilhelmsen, Gonneke Willemsen, Quenna Wong, Zhu-Ming Zhang, Alan B. Zonderman, Daniele Cusi, Michele K. Evans, Halina K. Greiser, Pim van der Harst, Mohammad Hassan, Erik Ingelsson, Marjo-Riitta Järvelin, Stefan Kääb, Mika Kähönen, Mika Kivimaki, Charles Kooperberg, Diana Kuh, Terho Lehtimäki, Lars Lind, Caroline M. Nievergelt, Chris J. O'Donnell, Albertine J. Oldehinkel, Brenda Penninx, Alexander P. Reiner, Harriëtte Riese, Arie M. van Roon, John D. Rioux, Jerome I. Rotter, Tamar Sofer, Bruno H. Stricker, Henning Tiemeier, Tanja G.M. Vrijkotte, Folkert W. Asselbergs, Bianca J.J.M Brundel, Susan R. Heckbert, Eric A. Whitsel, Marcel den Hoed, Harold Snieder \& Eco J.C. de Geus 